\title{
Improved Parametric Models and DPCM Techmiques for ECG Data Compression
}

\author{
Budagavi Madhukar", L.M. Patnaik* , I.S.N. Murthy* \\ *Dept. of Elec. Engineering **Dept. of Computer Sc. and Automation \\ Indian Institute of Science, Bangalore - 560012 , INDIA
}

\begin{abstract}
This paper presents a novel algorith for conpression of single lead blectrocardiogran (ECG) signals. The nethod is based on pole-zero nodelling of the Diacrete Cosine Transforwed (DCr) signal. An extension is proposed to the well known steiglitz-Mcbride algorithe, to model the higher frequency components of the input signal wore accurately. This is achieved by weighting the exror function miniaized by the algorith to estimate the codel paraneters. The data compression achieved by the paranetric model is further enhanced by Differential Pulse Code Modulation (DPCH) of the model paraneters. The nethod acconplishes a compression ratio in the range of $1: 20$ to $1: 40$, which far exceeds those achieved by most of the current nethods.
\end{abstract}

\section{Introduction}

Data Compression of ECG signals has been widely studied, in digital storage and transmission applications [1]. More recently the wide spread use of ambulatory ECG monitors has once again triggered a spur of research activities in this area. In this paper we propose an innovative algorithm for the compression of ECG signals based on the parametric modelling algorithm presented in [2]. The algorithm consisted of modelling the Discrete Cosine Transform (DCT) of the ECG signal using the Steiglitz-McBride (SM) method[3]. However, since the model impulse response died down quickly after excitation, it failed to reconstruct the higher DCT coefficients accurately. As DCT is a frequency transformation, loss of higher DCT coefficients results in loss of clinically significant notches such as the $Q$ and $S$ waves in some normal signals. In this paper we propose an extension to the SM method, to overcome this problem by weighting the error function. We call this technique as the Weighted SM method.

\section{I Alcorithim}

1) The Weighted Steiglitz-HcBride method: Let $S(k), k=0,1, \ldots,(N-1)$ be the DCT of the ECG signal. S( $\mathrm{k})$ is approximated as the impulse response of a model with a rational transfer function of the form,

$$
H(z)=\frac{N(z)}{D(z)}=B_{0} \frac{\left(1+B_{1} z^{-1}+\ldots+B_{p} z^{-p}\right)}{\left(1+A_{1} z^{-1}+\ldots+A_{p} z^{-D}\right)}
$$

where $p$ is the model order. In the original SM method, the model parameters $A_{i}$ and $B_{i}$ 0-7803-0785-2/92\$03.00 CIEEE are estimated by minimizing the sum of squared error between $S(k)$ and the model response, Here we weigh this error criterion by a function $w(k)$, to get

$$
E=\sum_{k=0}^{N-1} w(k)\left[S(k)-\frac{N(z)}{D(z)} \delta(k)\right]^{2}
$$

where $\delta(\mathrm{k})$ is the unit impulse. Minimization of $E$ in (2) with respect to the unknowns $A_{i}$ and $B_{i}$, results in a set of nonlinear equations. This problem is overcome by iteratively minimizing a similar error function $E^{\prime}[3]$, which is given by

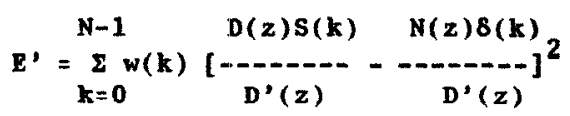

The algorithn is started with an AR estimate $D^{3}(z)$ obtained from linear prediction (LP) method and is continued till the difference in model parameters between two successive iterations is less than a prespecified threshold. In case of actual ECG data the algorithm always converged within 10 to 15 iterations.

2) Selection of weighting function: The DCT of the ECG signal contains most of its energy in the first 50 or so coefficients. Since most of the error energy minimized by the original SM algorithm is concentrated along the first few samples, the model impulse response matches $\mathrm{S}(\mathrm{k})$ closely only in this region. Extensive tests done have shown that for error free recovery of $Q$ and $S$ waves, accurate reconstruction of the DCT in the region from about $0.15 \mathrm{~N}$ to $0.6 \mathrm{~N}$ is also necessary. Thus we choose an error weighting function with larger gain in this region as shown in Fig 1 . The region $0.6 \mathrm{~N}$ onwards is weighted less as it contributes more to the noise in the signal than to any component waves.

3) Quantization: It is well known that quantization of the $M A$ and $A R$ coefficients in (1) needs a larger number of bits than that required to quantize the model zeros and poles, for the same quantization error. Thus the magnitude and phase of the model singularities viz. the poles and zeros are quantized along with the gain $B_{0}$ of the MA polynomial. Additional bits are used to indicate if the quantized singularities are 
real or in complex conjugate form, in which case only half the number of parameters are stored. The ECG signals were reconstructed with less than $7 \%$ NMSE when a zero, a pole and the gain were quantized with $24,22,14$ bits respectively.

4) Reconstruction: The ECG signal is reconstructed by computing the Inverse Discrete Cosine Transform (IDCT) of the model impulse response.

\section{Application of DPCM tBChNiques}

Using a simple QRS detection algorithm, we choose the frame of analysis to contain a beat of ECG signal. Due to the high degree of similarity between beats, the model parameters viz. poles and zeros, of adjacent frames change very little. We exploit this result to further enhance the bit rate by DPCM coding the poles and zeros of the consecutive frames. Redundancies in the information are removed by coding the difference between parameters of the two adjacent frames. This resulted in a substantial savings in the number of bits, by as much as $90 \%$.

\section{Resulirs}

The database for analysis consisted of 10 seconds of three lead ECG data, sampled from 60 subjects at $500 \mathrm{~Hz}$ and 12 bit resolution. Twelve of the subjects had rhythm disorders, 38 of them had morphological abnormalities while the remaining 10 were normal.

The original ECG signal, the signals

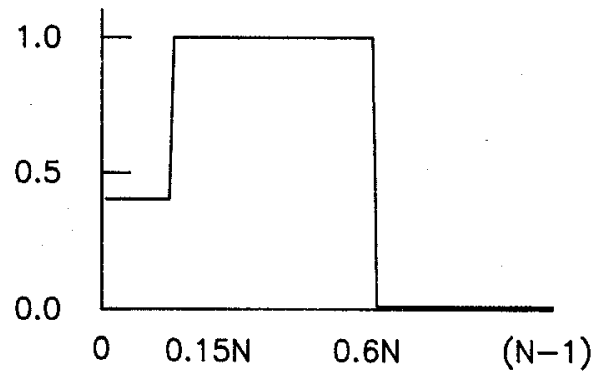

Fig. 1 The Weighting function w(k)

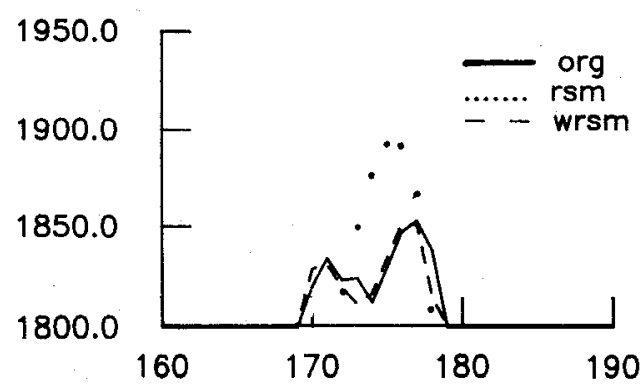

Fig. 2b Expanded view of boxed region of Fig. $2 a$ ( $X$ axis in all the Figures is sample number) reconstructed from the SM and WSM methods are shown in $\mathrm{Fig} 2 \mathrm{a}$. The expanded views of the corresponding QRS regions are shown in Fig $2 b$. As can be seen clearly the WSM has been able to model the notch while the SM has failed to do so. A model order of 10 was used for this signal.

Continuous records of normal sinus rhythm and the corresponding reconstructed signal are shown in Fig 3 . The signal was split into beat synchronous frames and modelled. The model order used was 10. DPCM of the model parameters resulted in a compression of $1: 35$. The NMSE of the reconstructed signal is $6 \%$.

\section{Conchusion}

We proposed a new method for efficient representation of ECG by modifying the error criterion used in identifying the signal model. The clinically significant $Q$ and $S$ waves and QRS notches were reconstructed with a high degree of accuracy. Use of DPCM on model parameters improved the compression from about $1: 20$ to $1: 40$.

\section{REFRRENCES}

[1] S.M.S. Jalaleddine, C.G. Hutchens, R.D. Strattan and W.A. Cobberly, "LCG data compression techniques - A unified approach," IEEE Trans. Bioned. Eng., Vol. 37, pp.329-343,April 1990.

[2] I.S.N. Murthy and G.S.S.D. Prasad, "Analysis of ECG from Pole-zero models," IREE Trans. Biomed. Eng., in press.

[3] $K$. Steiglitz and L. $\mathrm{L}$. McBride, "A technique for identification of linear systems," IEEE Trans. Automat. Contr. Vol 10, pp 461-464, Oct. 1965

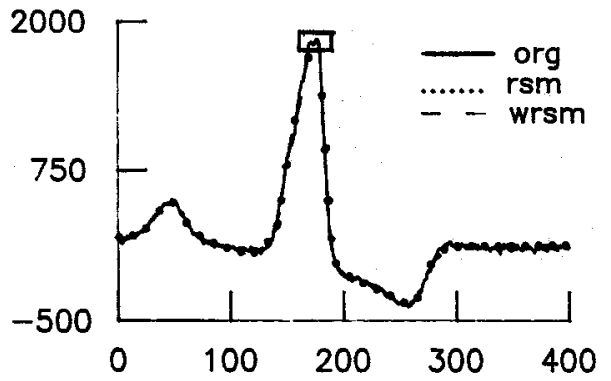

Fig. 2a The original Ecc(org) and the reconstructed BCGs using SH(rse) and WSH(wrsa)

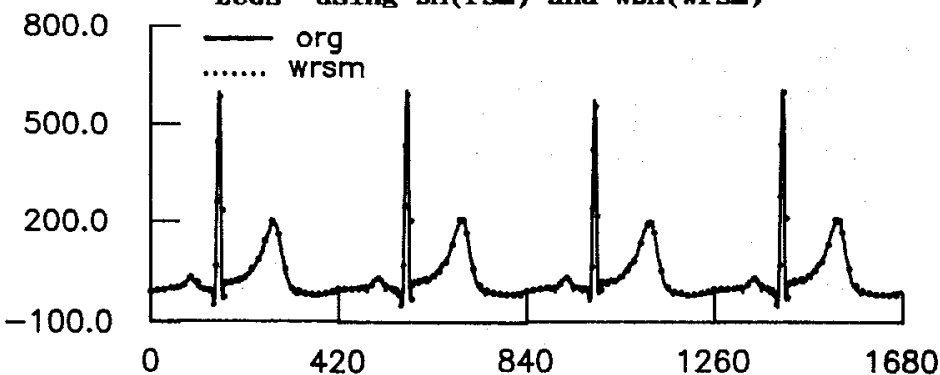

Fig. 3 Original rCG(org) and reconstructed ece(wram) ( $Y$ axis in all the Figures is amplitude) 